\title{
The Effects of Minimal Length in Entropic Force Approach
}

\author{
Barun Majumder \\ Indian Institute of Technology Gandhinagar, Ahmedabad, Gujarat 382424, India \\ Correspondence should be addressed to Barun Majumder; barunbasanta@iitgn.ac.in
}

Received 17 August 2013; Accepted 3 October 2013

Academic Editor: George Siopsis

Copyright (C) 2013 Barun Majumder. This is an open access article distributed under the Creative Commons Attribution License, which permits unrestricted use, distribution, and reproduction in any medium, provided the original work is properly cited.

\begin{abstract}
With Verlinde's recent proposal which says that gravity can be identified with an entropic force and considering the effects of generalized uncertainty principle in the black hole entropy-area relation we derive the modified equations for Newton's law of gravitation, modified Newtonian dynamics, and Einstein's general relativity. The corrections to the Newtonian potential is compared with the corrections that come from Randall-Sundrum II model and an effective field theoretical model of quantum general relativity. The effect of the generalized uncertainty principle introduces a $\sqrt{\text { area }}$ type correction term in the entropy-area relation whose consequences in different scenarios are discussed.
\end{abstract}

\section{Introduction}

One of the greatest achievements in theoretical physics is the realization that black holes are well-defined thermodynamic objects with entropy and temperature [1-5]. Hawking $[4,5]$ has derived that a Schwarzschild black hole emits a thermal radiation whose temperature depends on the mass $M$ of the black hole and is given by $T=1 / 8 \pi M$. Also Bekenstein has shown that a black hole has a well-defined entropy and is proportional to the area of the black hole horizon given by the entropy-area relation

$$
S_{\mathrm{BH}}=\frac{A}{4 l_{p}^{2}} \text {. }
$$

Here $A$ is the cross-sectional area of the black hole horizon and $l_{p}$ is the Planck length. Recently there has been much interest devoted to the leading order quantum corrections of the black hole entropy-area relation. Entropy accounts for the number of microstates of the system as it has a definite statistical meaning in thermodynamics. Sakharov is the originator of the idea of emergent gravity [6]. Jacobson [7] was the first to view Einstein's equation as an equation of state. Together with the second law of thermodynamics and the fact that entropy is proportional to the horizon area he derived the Einstein's equations. Later several studies were carried out to understand the deeper underlying connection between horizon thermodynamics and Einstein's equation.
Padmanabhan showed that for a wider class of theories the gravitational field equations on the horizon can be reduced to the first law of thermodynamics arguing the Einstein's equation to be a thermodynamic entity [8]. This novel idea was also later introduced in modified theories of gravity [9]. For a brief review on the demonstration of the idea in other scenarios we refer to [10-16]. The development in the lines discussed here refers to the point that thermodynamic properties can be associated with the horizon and gravity can be thought of as an entity whose origin is statistical in nature.

Recently Verlinde [17] introduced a very interesting proposal to understand the thermodynamic origin of gravity. According to him the changes in information which is associated with material bodies is the prime cause of gravity which is an entropic force. This even demands an explanation of the Newton's law of inertia and the equivalence principle may suggest that the origin of the law of inertia is entropic in nature. In his approach Newton's second law of motion can be recovered if one considers the idea of entropic force which is an effective macroscopic force which originates due to the statistical tendency of the increase in entropy. Also we have to consider the Unruh temperature which is the temperature experienced by an observer in an accelerated frame $\left(T=\hbar a / 2 \pi k_{B} c\right)$. Another observation is the recovery of the Newton's law of gravitation and its relativistic generalization to the Einstein's equation. For that the approach considers the idea of entropic force along with the equipartition of energy 
and the holographic principle. Though a thermodynamic interpretation of gravity can be given with the equipartition, argument even in nonrelativistic limit was established earlier [18]. Many authors recently focused on the understanding of the entropic force and [19-22] outline the literature.

In Verlinde's formalism he defined the Newton's constant $G$ through the relation

$$
N=\frac{A c^{3}}{G \hbar},
$$

where $N$ is the total number of bits and this relation follows from the holographic principle. Although he showed that this $G$ can actually be related to the Newton's constant, following the holographic principle it is a natural assumption that the number of bits is proportional to the area $A$. In a theory of emergent space area is defined in this form. Now as $l_{p}^{2}=$ $G \hbar / c^{3}$ where $l_{p}$ is the Planck length we get $N=A / l_{p}^{2}$. As the Bekenstein-Hawking entropy-area relation is $S_{\mathrm{BH}}=A / 4 l_{p}^{2}$ we have $N=4 S$.

Different theories of quantum gravity (e.g., [23-29]) have predicted the following form for the entropy of a black hole:

$$
S=\frac{A}{4 l_{p}^{2}}+c_{0} \ln \left(\frac{A}{4 l_{p}^{2}}\right)+\text { const. }
$$

$c_{0}$ is a model dependent parameter and $l_{p}$ is the Planck length. The speculation that the Heisenberg's uncertainty principle could be affected by the presence of gravity was done by Mead [30]. In the strong gravity regime, conventional Heisenberg uncertainty relation is no longer satisfactory (though approximately). Later modified commutation relations between position and momenta commonly known as the generalized uncertainty principle (GUP) were proposed by string theory, doubly special relativity (DSR), and black hole physics with the prediction of a minimum measurable length [31-40]. Similar kind of modification can also be found in the context of polymer quantization in terms of polymer mass scale [41]. Importance of the GUP can also be realized on the basis of simple gedanken experiments without any reference to a particular fundamental theory [38, 39]. So the GUP can be thought of as a model independent proposal, ideally suitable for the investigation of black hole entropy. The authors in $[42,43]$ proposed a GUP which is consistent with DSR, string theory, and black hole physics. This GUP is approximately covariant under DSR transformations but not Lorentz covariant [40]. With the GUP as proposed in [42, 43] we can arrive at the corrected entropy-area relation for a black hole which can be written as [44-46]

$$
\begin{aligned}
S \simeq & \frac{A}{4 l_{p}^{2}}+\alpha \sqrt{\frac{A}{4 l_{p}^{2}}}+\beta \ln \left(\frac{A}{4 l_{p}^{2}}\right) \\
& +\sum_{m=1 / 2,3 / 2, \ldots}^{\infty} \gamma_{m}\left(\frac{A}{4 l_{p}^{2}}\right)^{-m}+\sum_{n=1,2, \ldots}^{\infty} \delta_{n}\left(\frac{A}{4 l_{p}^{2}}\right)^{-n}+\text { const. }
\end{aligned}
$$

This is by far the most general form of quantum corrected entropy-area relation. In [44] black hole thermodynamics was first studied with modified dispersion relations and generalized uncertainty principle. Recently, many authors have suggested [47-52] that the GUP implications can be measured directly in tabletop experiments which will definitely confirm the theoretical predictions of some models. We can get some experimental bound on the deformation parameters $\alpha$ and $\beta$.

So in this paper we will study the effect of this corrected entropy-area relation in the theory of modified Newtonian dynamics (MOND). We will also follow Verlinde's viewpoint to construct the modified Newton's law and Einstein's equation with the entropy corrected relation of (4). $\alpha$ and $\beta$ are model dependent parameters in (4) and there are some predicted signs and values for $\alpha$ and $\beta$. But here we will consider a general treatment without concentrating on the values for the parameters. A similar approach was carried out by authors in $[53,54]$ where they considered logarithmic correction to the entropy-area relation $[23,27,29]$ and the power law corrections $[55,56]$. In [57] the effect of GUP on the Newtons law is studied in a different approach.

\section{Entropic Corrections due to GUP and the Modified Newtonian Dynamics (MOND)}

In 1983 Milgrom [58-60] gave a proposal to modify the Newtonian dynamics (commonly known as MOND) which can act as an alternative to nonbaryonic dark matter. After realizing the mass discrepancies in the galaxy rotation curves he proposed that for acceleration smaller than $1.2 \times 10^{-10} \mathrm{~m} / \mathrm{s}^{2}$ Newtonian dynamics needs a modification. Asymptotically the acceleration due to gravity is $a=\sqrt{a_{N} a_{0}}$, where $a_{N}$ is the Newtonian acceleration and $a_{0}=1.2 \times 10^{-10} \mathrm{~m} / \mathrm{s}^{2}$. MOND cannot be tested within the solar system as the strong gravitational field of the Sun dictates the dynamics. Usually the MOND acceleration due to gravity $a$ is written as

$$
a_{N}=a \mu\left(\frac{a}{a_{0}}\right) .
$$

In the asymptotic limit the interpolation function $\mu\left(a / a_{0}\right)$ admits $\mu=1$ for $a \gg a_{0}$ and $\mu=a / a_{0}$ for $a \ll a_{0}$ for the recovery of the Newtonian dynamics in the regime where the field is strong enough. For a review [61] is useful.

In the context of Verlinde's formalism, gravity theories have been connected with models of solid state physics, like the Debye's model at low temperature [62]. In [63] the onedimensional Debye model is shown to give MOND. Some recent attention also includes the derivation of MOND from the holographic entropy-area relation [53] and the collective motion of holographic screen bits [64]. Here bits are related to the units of information on the holographic screen. In the critical phenomena of cooling it can be shown that if in the equipartition relation, the zero energy bits are removed from the total number, and then we can get the notion of MOND. But we have to consider a modified equipartition theorem with the assumption that the division of energy is not homogeneous on all bits below a critical temperature. Then along with the holographic principle and the Unruh 
temperature we can recover the theory of MOND [65]. In the language of critical phenomena this is analogous to the first order phase transition. Following the methods of [65] we consider the fraction of bits with zero energy with

$$
N_{0}=N\left(1-\frac{T}{T_{c}}\right) .
$$

So for $T \geq T_{c}$ there are no bits with zero energy and the zero energy phenomena start for $T<T_{c}$. This is a relation for critical phenomena in second order phase transition. The number of bits with different energy at $T<T_{c}$ is given by

$$
N-N_{0}=N\left(\frac{T}{T_{c}}\right) .
$$

With the equipartition law of energy we get

$$
E=\frac{1}{2}\left(N \frac{T}{T_{c}}\right) T
$$

where we have considered $k_{B}=1$. Now with $E=M c^{2}$ we get

$$
T^{2}=\frac{2 M c^{2} T_{c}}{N},
$$

where $M$ is the emergent mass which can be considered to be at the center of the space enclosed by the holographic screen. Now we have the Unruh temperature $(T=\hbar a / 2 \pi c)$ which is associated with the acceleration of the frame and with (9) we get

$$
N a^{2}=\frac{8 \pi^{2} c^{2}}{\hbar^{2}} M c^{2} T_{c} .
$$

We have discussed earlier how entropy is related to $N$ as entropy is proportional to the number of bits. Here we use the entropy corrected relation of (4) and modifying $N=4 S$ we write

$$
\begin{aligned}
N= & \frac{A}{l_{p}^{2}}+4 \alpha \sqrt{\frac{A}{4 l_{p}^{2}}}+4 \beta \ln \frac{A}{4 l_{p}^{2}} \\
& +4 \gamma\left(\frac{A}{4 l_{p}^{2}}\right)^{-1 / 2}+4 \delta\left(\frac{A}{4 l_{p}^{2}}\right)^{-1} .
\end{aligned}
$$

With $A=4 \pi R^{2}$ and using (11) we rewrite (10) as

$$
\begin{aligned}
a^{2}\left(\frac{4 \pi R^{2}}{l_{p}^{2}}\right)\left[1+\frac{\alpha l_{p}}{\sqrt{\pi} R}+\frac{\beta l_{p}^{2}}{\pi R^{2}} \ln \left\{\frac{\pi R^{2}}{l_{p}^{2}}\right\}\right. \\
\left.+\frac{\gamma l_{p}^{3}}{\pi^{3 / 2} R^{3}}+\frac{\delta l_{p}^{4}}{\pi^{2} R^{4}}\right]=\frac{8 \pi^{2} c^{2}}{\hbar^{2}} M c^{2} T_{c} .
\end{aligned}
$$

We also mention that we considered only the leading order terms in the entropy-area relation of (4). With a little algebra and considering $a_{0}=(2 \pi c / \hbar) T_{c}$ we can finally arrive at

$$
\begin{aligned}
a\left(\frac{a}{a_{0}}\right)=\frac{G M}{R^{2}} & {\left[1-\frac{\alpha l_{p}}{\sqrt{\pi} R}-\frac{\beta l_{p}^{2}}{\pi R^{2}} \ln \left\{\frac{\pi R^{2}}{l_{p}^{2}}\right\}\right.} \\
& \left.-\frac{\gamma l_{p}^{3}}{\pi^{3 / 2} R^{3}}-\frac{\delta l_{p}^{4}}{\pi^{2} R^{4}}\right] .
\end{aligned}
$$

Here we have only first order terms of $\alpha, \beta, \gamma$, and $\delta$. This equation is the entropy corrected equation for the modified Newtonian dynamics.

\section{Entropic Corrections due to GUP and the Newton's Law of Gravitation}

Bekenstein's entropy-area relation [3] came from the argument that if a particle is within the Compton wavelength from a black hole horizon then it is a part of the black hole. There will be an increase in mass and area of the black hole and the relevant change is identified with one bit of information. With this motivation Verlinde postulated that the entropy associated with the information at the boundary is given by

$$
\Delta S=2 \pi \quad \text { when } \Delta x=\frac{\hbar}{m c} \text {. }
$$

Here we have considered $k_{B}=1$. If we assume that the change in entropy is linear to $\Delta x$ then we can rewrite (14) as

$$
\Delta S=2 \pi \frac{m c}{\hbar} \Delta x
$$

This idea is analogous to osmosis across a semipermeable membrane. As the membrane carries a temperature $T$ so the particle will experience an effective entropic force

$$
F \Delta x=T \Delta S \text {. }
$$

This force is attractive. A nonzero force leads to a nonzero acceleration and acceleration is related to temperature by Unruh effect. If we now assume that the total energy $E$ of the system is divided evenly over $N$ bits then the temperature is given by the equipartition law of energy

$$
T=\frac{2 E}{N} .
$$

With $E=M c^{2}$ we get

$$
T=\frac{2 M c^{2}}{N}
$$

So with (15), (16), and (18) we have

$$
F=\frac{2 M c^{2}}{N} \frac{2 \pi m c}{\hbar} .
$$

As mentioned earlier we will study the entropy corrected version of this equation. So with the entropy corrections which are incorporated in $N$ (11) we can write the entropic force equation as

$$
\begin{gathered}
F=\frac{G M m}{R^{2}}\left[1-\frac{\alpha l_{p}}{\sqrt{\pi} R}-\frac{\beta l_{p}^{2}}{\pi R^{2}} \ln \left\{\frac{\pi R^{2}}{l_{p}^{2}}\right\}\right. \\
\left.-\frac{\gamma l_{p}^{3}}{\pi^{3 / 2} R^{3}}-\frac{\delta l_{p}^{4}}{\pi^{2} R^{4}}\right],
\end{gathered}
$$


with $A=4 \pi R^{2}$. If $\alpha=\beta=\gamma=\delta=0$ this is the Newton's law of gravitation. The Newtonian potential turns out to be

$$
\begin{aligned}
V(R) \sim \frac{G M m}{R}[ & 1-\frac{\alpha l_{p}}{2 \sqrt{\pi} R}-\frac{2 \beta l_{p}^{2}}{9 \pi R^{2}} \\
& \left.-\frac{\beta l_{p}^{2}}{3 \pi R^{2}} \ln \left(\frac{\pi R^{2}}{l_{p}^{2}}\right)+\sigma\left(l_{p}^{3}\right)\right] .
\end{aligned}
$$

This modification of Newton's law is similar to the predictions came from Randall-Sundrum II model [66]. The model has one uncompactified dimension with length scale $l_{\mu}$. But here the sign of the prefactor of the correction is different. If we would have considered the entropy corrected relation of [46] then this sign ambiguity would not have come. In the RS braneworld scenario the Newtonian potential is calculated as [67]

$$
V(r) \sim \begin{cases}\frac{1}{R}\left[1+\frac{4 l_{\mu}}{3 \pi R}-\cdots\right] & \text { for } l_{\mu} \gg R, \\ \frac{1}{R}\left[1+\frac{2 l_{\mu}^{2}}{3 R^{2}}-\cdots\right] & \text { for } l_{\mu} \ll R,\end{cases}
$$

where $l_{\mu}$ is the characteristic length scale of the theory. The significant prediction of [67] is that gravity is fivedimensional at short distances. This comparison of the modified Newton's law of gravitation with respect to the RS II model was first pointed out in [68]. The modified entropy-area relation that we have used is a consequence of the GUP and the question can be raised that whether the GUP modifications predict the same results as that of RS II model for short distance physics. Although Newton's $1 / R^{2}$ force law is the only law of gravitation up to $0.13 \mathrm{~mm}[69,70]$ it is still unknown whether the law is valid at much lower scales. Here it is possible to put an upper bound on $\alpha$ from the RS II characteristic length scale $l_{\mu}$. If the tension $1 / l_{\mu}$ of the brane is small enough compared to the Planck mass then the correction to the Newtonian potential would help us to distinguish RS II model from other extradimensional models. $l_{\mu}$ is constrained by present short distance tests of gravity which predict $l_{\mu}<11 \times 10^{-06} \mathrm{~m}[71,72]$. If we use this bound on $l_{\mu}$ and compare with the first order correction of (21) we get an upper bound on the deformation parameter $\alpha$ which is $<10^{29}$. This bound is not sensitive for phenomenological purposes as this intermediate length scale should be $\leq 10^{17}$; otherwise it would have been observed as the electroweak length scale is $\sim 10^{17} l_{p}[42,43]$. Also the current experimental bounds on $\alpha$ are $\leq 10^{17}, 10^{10}$, and $10^{11}$ from position measurement, Hydrogen Lamb shift, and electron tunneling, respectively $[47-50,73,74]$. On the other hand in $5 \mathrm{D}$ heterotic M-theory if the 5-dimensional fundamental mass is of the order of grand unification scale $\left(10^{16} \mathrm{GeV}\right)$ then the corrections to the Newtonian potential would be relevant at $l_{\mu} \sim 10^{-26} \mathrm{~m}$ [75]. Now correction of this order if compared to the first order correction of (21) would give an upper bound for the parameter $\alpha$ which is $<10^{9}$.
It is also interesting that if we consider the scattering of two heavy masses $m_{1}$ and $m_{2}$ in a gravitational potential, the nonrelativistic potential gets some corrections. We can write

$$
V(r) \sim \frac{G m_{1} m_{2}}{r}\left[1+\frac{3 G\left(m_{1}+m_{2}\right)}{r c^{2}}+\frac{41 l_{p}^{2}}{10 \pi r^{2}}\right] .
$$

This is the Donoghue potential [76-80]. The first correction term is the classical post-Newtonian correction and the last correction is purely quantum. For the derivation one has to treat quantum general relativity as an effective field theory. The last correction term of the Donoghue potential does not fit well for the phenomenological purposes but it definitely shows a well behaved classical limit. The first correction term of the potential is not considered as a quantum correction as for a small test particle $m_{2}$ this is similar to the time component $g_{00}$ of the Schwarzschild metric which is the source of the static gravitational potential [80]. In the process to get (21) Newton's law arose naturally with the consideration that space is emergent through a holographic scenario [17]. But (23) is an artifact of treating quantum general relativity as an effective field theory. Here also we see that the predictions of an emergent theory of gravity with the effects of the generalized uncertainty principle are similar to that of quantum general relativity. However, it is not surprising that any theory of quantum gravity comes with an intrinsic length scale and the low energy effective theory is plagued with corrections associated with the length scale.

\section{Entropic Corrections due to GUP and the Einstein's Equation}

In the earlier section we have considered nonhomogeneous cooling of bits which restricted the distribution of energy equally on all bits of the holographic screen below a critical temperature. In turn we get a modified equipartition law of energy and we derive MOND in presence of the effects of generalized uncertainty principle. In this section we further investigate the effects of the results derived earlier on the Einstein's field equations. With the assumption that the holographic principle holds and considering the fact that each single bit of information occupies a unit cell one can write

$$
N=\frac{A}{l_{p}^{2}}, \quad \text { where } l_{p}^{2}=\frac{G \hbar}{c^{3}}
$$

This is four times the Bekenstein-Hawking entropy which says $S_{\mathrm{BH}}=A / 4 l_{p}^{2}$. So that we can write

$$
N=4 S \text {. }
$$

Considering the entropic corrections due to GUP (11) we can write

$$
d N=\frac{1}{l_{p}^{2}}\left[1+\frac{\alpha l_{p}}{\sqrt{A}}+\frac{4 \beta l_{p}^{2}}{A}-\frac{4 \gamma l_{p}^{3}}{A^{3 / 2}}-\frac{16 \delta l_{p}^{4}}{A^{2}}\right] d A
$$


This is the bit density on the screen. If the energy associated with mass $M$ is divided over all bits and each bit carries mass $(1 / 2) T$ due to the equipartition law we have

$$
M=\frac{1}{2} \int_{S} T d N
$$

The local temperature $T$ on the screen is given by

$$
T=\frac{\hbar}{2 \pi} e^{\phi} n^{b} \nabla_{b} \phi
$$

where $e^{\phi}$ is the redshift factor as $T$ is measured from infinity. So (27) is written as

$$
M=\frac{1}{4 \pi G} \int_{S} e^{\phi} \nabla \phi \cdot\left[1+\frac{\alpha l_{p}}{\sqrt{A}}+\frac{4 \beta l_{p}^{2}}{A}-\frac{4 \gamma l_{p}^{3}}{A^{3 / 2}}-\frac{16 \delta l_{p}^{4}}{A^{2}}\right] d A,
$$

where we have considered $c=1$. This equation is the modified Gauss law in general relativity and the right hand side represents the modified Komar mass. The first integral of (29) is the Komar mass $M_{K}$ and

$$
M_{K}=\frac{1}{4 \pi G} \int_{S} e^{\phi} \nabla \phi \cdot d A
$$

Now this relation can be written in terms of the Ricci tensor $R_{a b}$ and the Killing vector $\xi^{a}[17,81]$ where one uses the Stokes theorem and the Killing equation for $\xi^{a}: \nabla^{a} \nabla_{a} \xi^{b}=-R_{a}^{b} \xi^{a}$. Finally one can get

$$
M_{K}=\frac{1}{4 \pi G} \int_{\Sigma} R_{a b} n^{a} \xi^{b} d V
$$

So we rewrite (29) as

$$
\begin{aligned}
M= & \frac{1}{4 \pi G} \int_{\Sigma} R_{a b} n^{a} \xi^{b} d V \\
& +\frac{l_{p}}{4 \pi G} \int_{S} e^{\phi} \nabla \phi \cdot\left[\frac{\alpha}{\sqrt{A}}+\frac{4 \beta l_{p}}{A}-\frac{4 \gamma l_{p}^{2}}{A^{3 / 2}}-\frac{16 \delta l_{p}^{3}}{A^{2}}\right] d A,
\end{aligned}
$$

where $\Sigma$ is the three-dimensional volume bounded by $S$ which is the holographic screen and $n^{a}$ is the normal. Also $M$ can be written as a volume integral of the stress energy tensor $T_{a b}$, where

$$
M=2 \int_{\Sigma}\left(T_{a b}-\frac{1}{2} T g_{a b}\right) n^{a} \xi^{b} d V
$$

So with (32) and (33) we can write the entropy corrected Einstein's equation as

$$
\begin{aligned}
& \int_{\Sigma}\left[R_{a b}-8 \pi G\left(T_{a b}-\frac{1}{2} T g_{a b}\right)\right] n^{a} \xi^{b} d V \\
& \quad=-l_{p} \int_{S} e^{\phi} \nabla \phi \cdot\left(\frac{\alpha}{\sqrt{A}}+\frac{4 \beta l_{p}}{A}-\frac{4 \gamma l_{p}^{2}}{A^{3 / 2}}-\frac{16 \delta l_{p}^{3}}{A^{2}}\right) d A .
\end{aligned}
$$

If $\alpha=\beta=\gamma=\delta=0$ we get the usual Einstein's equation. Here we have surface corrections which came as a consequence of the correction to the density of bits on the holographic screen. In a spherically symmetric static space time with a little algebra finally we can get the entropy corrected Einstein's equation as

$$
R_{a b}=8 \pi G\left(T_{a b}-\frac{1}{2} T g_{a b}\right)\left(1+\alpha^{\prime}\right),
$$

where

$$
\alpha^{\prime}=\frac{l_{p}}{2 \pi}\left(\frac{\alpha}{\sqrt{A}}+\frac{4 \beta l_{p}}{A}-\frac{4 \gamma l_{p}^{2}}{A^{3 / 2}}-\frac{16 \delta l_{p}^{3}}{A^{2}}\right) .
$$

For large horizon area the equation reduces to usual Einstein's equation.

\section{Discussion}

Gravity may be identified to be associated with entropic force and a thermodynamical system may well describe a gravitational system. This idea came from the thermodynamical interpretation of gravitational field equations. A holographic screen is assumed to contain the information of the volume enclosed by it and the information is divided in bits. So according to Verlinde it is natural to assume that the number of bits is proportional to the area of the holographic screen. On the other hand, all approaches to quantum gravity support the idea of existence of a minimal observable length of the order (or some order) of Planck length. Also it is conjectured that the standard commutation relations at short distances would be modified. In $[42,43]$ a form of the generalized uncertainty principle was proposed which is consistent with doubly special relativity (DSR), string theory, and black holes physics with the prediction of a maximum observable momentum and a minimal measurable length. As an immediate effect of the quantum gravity corrections incorporated through the GUP the entropy-area relation of a black hole gets modified. So it leads to a modification in the number of bits of information on the holographic screen as discussed in Verlinde's approach. Verlinde's approach is found to be consistent if one obtains modifications to the Newton's law from the log corrected entropy-area relation as the modifications have the same form as that of the lowest order quantum corrections of perturbative quantum gravity [53].

In this paper we have generalized the entropic force law as introduced by Verlinde via a phenomenological interpretation of the generalized uncertainty principle. Considering the effects of generalized uncertainty principle in the black hole entropy-area relation here we have derived the modified equations for Newton's law of gravitation, modified Newtonian dynamics, and Einstein's general relativity. The leading order correction of the modified potential in the Newton's law of gravitation surprisingly agrees with the short distance Newtonian potential as predicted by Randall-Sundrum II model. As the RS II model has an uncompactified extra dimension so it would be interesting to investigate whether 
the GUP effects can predict the same as that of the extra dimensional theories. It is also interesting to note that the corrections to the Newtonian potential which we get are similar to the Donoghue potential which is a consequence of treating quantum general relativity as an effective field theory. We found that the corrections due to an emergent theory of gravity with the effects of minimal length are similar to that of quantum general relativity. This is quite evident as any theory of quantum gravity is accompanied by an intrinsic length scale which manifests itself as the coefficient of leading order corrections in low energy phenomena. Here with Verlinde's approach we observe that the GUP motivated entropy-area relation modifies Newton's law with modifications that are similar to different order of quantum effects as evidenced in perturbative quantum gravity. Later we derived the modified Einstein's field equation in the same framework which for large horizon areas reduces to the usual Einstein's field equation.

\section{Acknowledgments}

The author would like to acknowledge the support of APSIUSSTF Visitation Program as a part of the present work that was completed during the visit. The author would also like to thank an anonymous referee for enlightening comments and helpful suggestions.

\section{References}

[1] J. D. Bekenstein, "Black holes and the second law," Lettere Al Nuovo Cimento, vol. 4, no. 15, pp. 737-740, 1972.

[2] J. D. Bekenstein, "Generalized second law of thermodynamics in black-hole physics," Physical Review D, vol. 9, no. 12, pp. 32923300, 1974.

[3] J. D. Bekenstein, "Black holes and entropy," Physical Review D, vol. 7, no. 8, pp. 2333-2346, 1973.

[4] S. W. Hawking, "Black hole explosions?" Nature, vol. 248, no. 5443, pp. 30-31, 1974.

[5] S. W. Hawking, "Particle creation by black holes," Communications in Mathematical Physics, vol. 43, pp. 199-220, 1975.

[6] A. D. Sakharov, "Vacuum quantum fluctuations in curved space and the theory of gravitation," General Relativity and Gravitation , vol. 32, no. 2, pp. 365-367, 2000, Translated from Doklady Akademii Nauk SSSR, vol. 177, No. 1, pp. 7071, November 1967.

[7] T. Jacobson, "Thermodynamics of spacetime: the Einstein equation of state," Physical Review Letters, vol. 75, no. 7, pp. 1260-1263, 1995.

[8] T. Padmanabhan, "Classical and quantum thermodynamics of horizons in spherically symmetric spacetimes," Classical and Quantum Gravity, vol. 19, no. 21, pp. 5387-5408, 2002.

[9] C. Eling, R. Guedens, and T. Jacobson, "Nonequilibrium thermodynamics of spacetime," Physical Review Letters, vol. 96, no. 12, Article ID 121301, 2006.

[10] T. Padmanabhan, "Thermodynamical aspects of gravity: new insights," Reports on Progress in Physics, vol. 73, no. 4, Article ID 046901, 2010.

[11] M. Akbar and R. G. Cai, "Thermodynamic behavior of the Friedmann equation at the apparent horizon of the FRW universe," Physical Review D, vol. 75, no. 8, Article ID 084003, 9 pages, 2007.

[12] R. G. Cai and S. P. Kim, "First law of thermodynamics and Friedmann equations of Friedmann-Robertson-Walker universe," Journal of High Energy Physics, vol. 02, p. 050, 2005.

[13] A. Sheykhi, B. Wang, and R. G. Cai, "Thermodynamical properties of apparent horizon in warped DGP braneworld," Nuclear Physics B, vol. 779, no. 1-2, pp. 1-12, 2007.

[14] R. G. Cai and L. M. Cao, "Thermodynamics of apparent horizon in brane world scenario," Nuclear Physics B, vol. 785, no. 1-2, pp. 135-148, 2007.

[15] A. Sheykhi, B. Wang, and R. G. Cai, "Deep connection between thermodynamics and gravity in Gauss-Bonnet braneworlds," Physical Review D, vol. 76, Article ID 023515, 5 pages, 2007.

[16] A. Sheykhi and B. Wang, "Generalized second law of thermodynamics in Gauss-Bonnet braneworld," Physics Letters B, vol. 678, no. 5, pp. 434-737, 2009.

[17] E. P. Verlinde, "On the origin of gravity and the laws of Newton," Journal of High Energy Physics, vol. 2011, article 29, 2011.

[18] T. Padmanabhan, "Equipartition of energy in the horizon degrees of freedom and the emergence of gravity," Modern Physics Letters A, vol. 25, no. 14, pp. 1129-1136, 2010.

[19] L. Smolin, "Newtonian gravity in loop quantum gravity," http:// arxiv.org/abs/1001.3668.

[20] R. G. Cai, L. M. Cao, and N. Ohta, "Friedmann equations from entropic force," Physical Review D, vol. 81, no. 6, Article ID 061501(R), 4 pages, 2010.

[21] A. Sheykhi, "Entropic corrections to Friedmann equations," Physical Review D, vol. 81, no. 10, Article ID 104011, 4 pages, 2010.

[22] A. Sheykhi and S. H. Hendi, "Power-law entropic corrections to Newton's law and Friedmann equations," Physical Review D, vol. 84, no. 4, Article ID 044023, 8 pages, 2011.

[23] R. K. Kaul and P. Majumdar, "Logarithmic correction to the Bekenstein-Hawking entropy," Physical Review Letters, vol. 84, no. 23, pp. 5255-5257, 2000.

[24] A. J. M. Medved and E. C. Vagenas, "When conceptual worlds collide: the generalized uncertainty principle and the Bekenstein-Hawking entropy," Physical Review D, vol. 70, no. 12, Article ID 124021, 5 pages, 2004.

[25] S. Das, P. Majumdar, and R. K. Bhaduri, "General logarithmic corrections to black-hole entropy," Classical and Quantum Gravity, vol. 19, no. 9, p. 2355, 2002.

[26] M. Domagala and J. Lewandowski, "Black-hole entropy from quantum geometry," Classical and Quantum Gravity, vol. 21, no. 22, p. 5233, 2004.

[27] A. Chatterjee and P. Majumdar, "Universal canonical black hole entropy," Physical Review Letters, vol. 92, no. 14, Article ID 141301, 2004.

[28] G. Amelino-Camelia, M. Arzano, and A. Procaccini, "Severe constraints on the loop-quantum-gravity energy-momentum dispersion relation from the black-hole area-entropy law," Physical Review D, vol. 70, no. 10, Article ID 107501, 2004.

[29] K. A. Meissner, "Black-hole entropy in loop quantum gravity," Classical and Quantum Gravity, vol. 21, no. 22, pp. 5245-5251, 2004.

[30] C. A. Mead, "Possible connection between gravitation and fundamental length," Physical Review, vol. 135, no. 3B, pp. B849B862, 1964. 
[31] D. Amati, M. Ciafaloni, and G. Veneziano, "Can spacetime be probed below the string size?" Physics Letters B, vol. 216, no. 1-2, pp. 41-47, 1989.

[32] M. Maggiore, "The algebraic structure of the generalized uncertainty principle," Physics Letters B, vol. 319, no. 1-3, pp. 83-86, 1993.

[33] S. Hossenfelder, M. Bleicher, S. Hofmann, J. Ruppert, S. Scherer, and H. Stöcker, "Signatures in the Planck regime," Physics Letters $B$, vol. 575, no. 1-2, pp. 85-99, 2003.

[34] C. Bambi and F. R. Urban, "Natural extension of the generalized uncertainty principle," Classical and Quantum Gravity, vol. 25, no. 9, Article ID 095006, 2008.

[35] A. Kempf, G. Mangano, and R. B. Mann, "Hilbert space representation of the minimal length uncertainty relation," Physical Review D, vol. 52, no. 2, pp. 1108-1118, 1995.

[36] F. Brau, "Minimal length uncertainty relation and the hydrogen atom," Journal of Physics A, vol. 32, no. 44, pp. 7691-7696, 1999.

[37] J. Magueijo and L. Smolin, "Lorentz invariance with an invariant energy scale," Physical Review Letters, vol. 88, no. 19, pp. 1904031-1904034, 2002.

[38] M. Maggiore, "A generalized uncertainty principle in quantum gravity," Physics Letters B, vol. 304, no. 1-2, pp. 65-69, 1993.

[39] F. Scardigli, "Generalized uncertainty principle in quantum gravity from micro-black hole gedanken experiment," Physics Letters B, vol. 452, no. 1-2, pp. 39-44, 1999.

[40] J. L. Cortes and J. Gamboa, "Quantum uncertainty in doubly special relativity," Physical Review D, vol. 71, no. 6, Article ID 065015, 4 pages, 2005.

[41] G. M. Hossain, V. Husain, and S. S. Seahra, "Backgroundindependent quantization and the uncertainty principle," Classical and Quantum Gravity, vol. 27, no. 16, Article ID 165013, 2010.

[42] A. F. Ali, S. Das, and E. C. Vagenas, "Discreteness of space from the generalized uncertainty principle," Physics Letters B, vol. 678, no. 5, pp. 497-499, 2009.

[43] S. Das and E. C. Vagenas, "Universality of quantum gravity corrections," Physical Review Letters, vol. 101, Article ID 221301, 4 pages, 2008.

[44] G. Amelino-Camelia, M. Arzano, Y. Ling, and G. Mandanici, "Black-hole thermodynamics with modified dispersion relations and generalized uncertainty principles," Classical and Quantum Gravity, vol. 23, no. 7, pp. 2585-2606, 2006.

[45] B. Majumder, "Black hole entropy and the modified uncertainty principle: a heuristic analysis," Physics Letters B, vol. 703, no. 4, pp. 402-405, 2011.

[46] B. Majumder, "Black hole entropy with minimal length in tunneling formalism," General Relativity and Gravitation, vol. 45, no. 11, pp. 2403-2414, 2013.

[47] I. Pikovski, M. R. Vanner, M. Aspelmeyer et al., "Probing Planck-scale physics with quantum optics," Nature Physics, vol. 8, pp. 393-397, 2012.

[48] I. Pikovski, M. R. . Vanner, M. Aspelmeyer, M. Kim, and C. Brukner, "Probing Planck-scale physics with quantum optics," Nature Physics, vol. 8, p. 393, 2012.

[49] A. F. Ali, S. Das, and E. C. Vagenas, "Proposal for testing quantum gravity in the lab," Physical Review D, vol. 84, Article ID 044013, p. 10, 2011.

[50] S. Das and E. C. Vagenas, "Universality of quantum gravity corrections," Physical Review Letters, vol. 101, Article ID 221301, 4 pages, 2008.
[51] J. D. Bekenstein, "Is a tabletop search for Planck scale signals feasible?” Physical Review D, vol. 86, Article ID 124040, 9 pages, 2012.

[52] J. D. Bekenstein, "Can quantum gravity be exposed in the laboratory? A tabletop experiment to reveal the quantum foam," http://arxiv.org/abs/1301.4322.

[53] L. Modesto and A. Randono, "Entropic corrections to Newton's law," http://arxiv.org/abs/1003.1998.

[54] S. H. Hendi and A. Sheykhi, "Entropic corrections to Einstein equations," Physical Review D, vol. 83, no. 8, Article ID 084012, 2011.

[55] S. Das, S. Shankaranarayanan, and S. Sur, "Power-law corrections to entanglement entropy of horizons," Physical Review D, vol. 77, no. 6, Article ID 064013, 2008.

[56] N. Radicella and D. Pavon, "The generalized second law in universes with quantum corrected entropy relations," Physics Letters B, vol. 691, no. 3, p. 126, 2010.

[57] K. Nozari, P. Pedram, and M. Molkara, "Minimal length, maximal momentum and the entropic force law," International Journal of Theoretical Physics, vol. 51, no. 4, pp. 1268-1275, 2012.

[58] M. Milgrom, "A modification of the Newtonian dynamics as a possible alternative to the hidden mass hypothesis," Astrophysical Journal, vol. 270, pp. 365-370, 1983.

[59] M. Milgrom, "A modification of the Newtonian dynamicsimplications for galaxies," Astrophysical Journal, vol. 270, pp. 371-389, 1983.

[60] M. Milgrom, "A modification of the Newtonian dynamicsimplications for galaxy systems," Astrophysical Journal, vol. 270, pp. 384-389, 1983.

[61] R. H. Sanders and S. S. McGaugh, "Modified Newtonian dynamics as an alternative to dark matter," Annual Review of Astronomy and Astrophysics, vol. 40, pp. 263-317, 2002.

[62] C. Gao, "Modified entropic force," Physical Review D, vol. 81, no. 8, Article ID 087306, 4 pages, 2010.

[63] X. Li and Z. Chang, "Debye entropic force and modified Newtonian dynamics," Communications in Theoretical Physics, vol. 55, no. 4, pp. 733-736, 2011.

[64] V. V. Kiselev and S. A. Timofeev, "The holographic screen at low temperatures," Modern Physics Letters A, vol. 26, no. 2, pp. 109118,2011

[65] J. A. Neto, "Nonhomogeneous cooling, entropic gravity and MOND theory," International Journal of Theoretical Physics, vol. 50, no. 11, pp. 3552-3559, 2011.

[66] L. Randall and R. Sundrum, "An alternative to compactification," Physical Review Letters, vol. 83, no. 23, pp. 4690-4693, 1999.

[67] P. Callin and F. Ravndal, "Higher order corrections to the Newtonian potential in the Randall-Sundrum model," Physical Review D, vol. 70, no. 10, Article ID 104009, pp. 1-104009, 2004.

[68] A. F. Ali and A. N. Tawfik, "Modified Newton's law of gravitation due to minimal length in quantum gravity," Advances in High Energy Physics, vol. 2013, Article ID 126528, 7 pages, 2013.

[69] S.-Q. Yang, B.-F. Zhan, Q.-L. Wang et al., "Test of the gravitational inverse square law at millimeter ranges," Physical Review Letters, vol. 108, no. 8, Article ID 081101, 5 pages, 2012.

[70] C. D. Hoyle, D. J. Kapner, B. R. Heckel et al., "Submillimeter tests of the gravitational inverse-square law," Physical Review D, vol. 70, no. 4, Article ID 042004, 31 pages, 2004.

[71] D. J. Kapner, T. S. Cook, E. G. Adelberger et al., "Tests of the gravitational inverse-square law below the dark-energy length 
scale," Physical Review Letters, vol. 98, no. 2, Article ID 021101, 2007.

[72] E. G. Adelberger, B. R. Heckel, S. Hoedl, C. D. Hoyle, D. J. Kapner, and A. Upadhye, "Particle-physics implications of a recent test of the gravitational inverse-square law," Physical Review Letters, vol. 98, no. 13, Article ID 131104, 4 pages, 2007.

[73] H. Grote, "The status of GEO 600," Classical and Quantum Gravity, vol. 25, no. 11, Article ID 114043, 2008.

[74] B. P. Abbott, R. Abbott, R. Adhikari et al., "LIGO: the laser interferometer gravitational-wave observatory," Reports on Progress in Physics, vol. 72, no. 7, Article ID 076901, 2009.

[75] G. A. Palma, "On Newton's law in supersymmetric braneworld models," Journal of High Energy Physics, vol. 2007, p. 091, 2007.

[76] J. F. Donoghue, "General relativity as an effective field theory: the leading quantum corrections," Physical Review D, vol. 50, p. $3874,1994$.

[77] N. E. J. Bjerrum-Bohr, J. F. Donoghue, and B. R. Holstein, "Quantum gravitational corrections to the nonrelativistic scattering potential of two masses," Physical Review D, vol. 67, Article ID 084033, 2003.

[78] I. B. Khriplovich and G. G. Kirilin, "Quantum power correction to the Newton law," Journal of Experimental and Theoretical Physics, vol. 95, pp. 981-986, 2002.

[79] J. F. Donoghue, “The effective field theory treatment of quantum gravity," http://arxiv.org/abs/1209.3511.

[80] J. F. Donoghue, "Leading quantum correction to the Newtonian potential," Physical Review Letters, vol. 72, no. 19, pp. 2996-2999, 1994.

[81] R. M. Wald, General Relativity, Chicago University Press, Chicago, Ill, USA, 1984. 

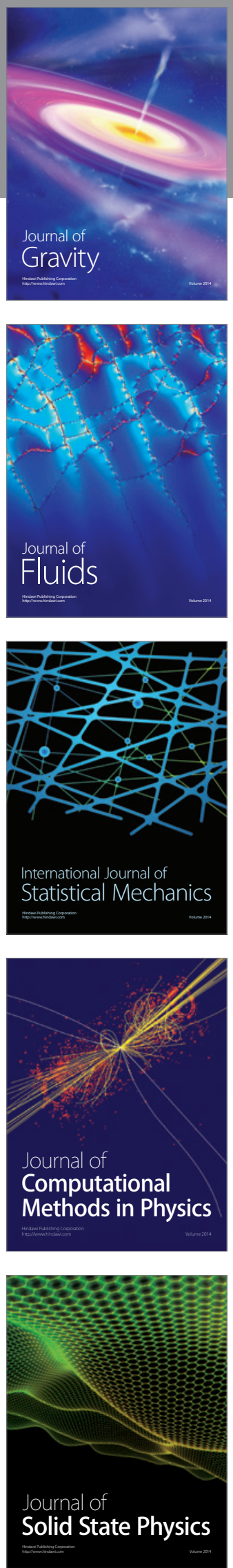

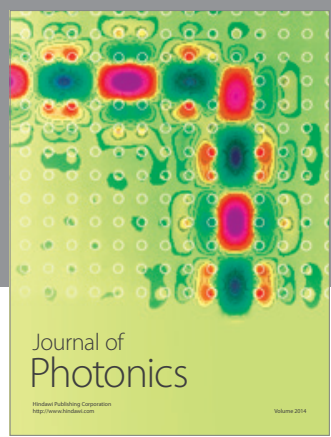

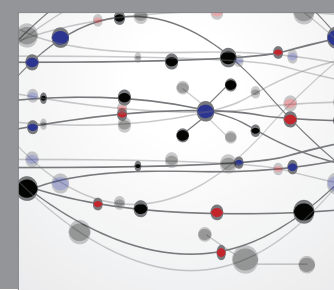

The Scientific World Journal

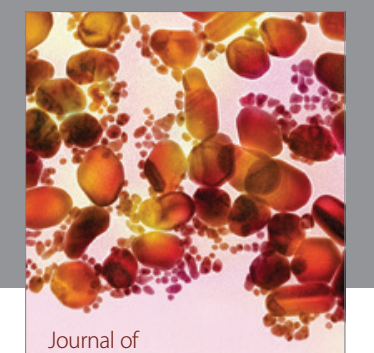

Soft Matter
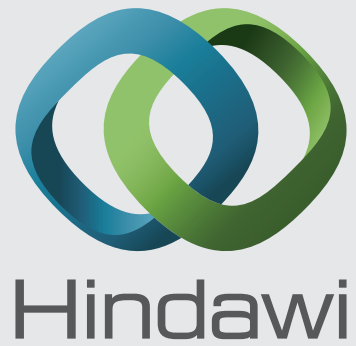

Submit your manuscripts at

http://www.hindawi.com
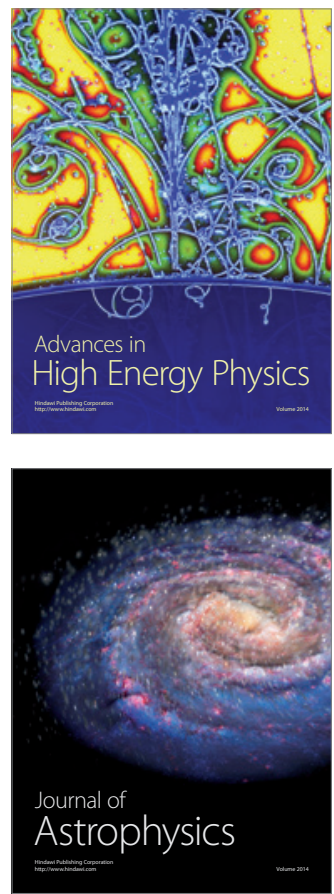
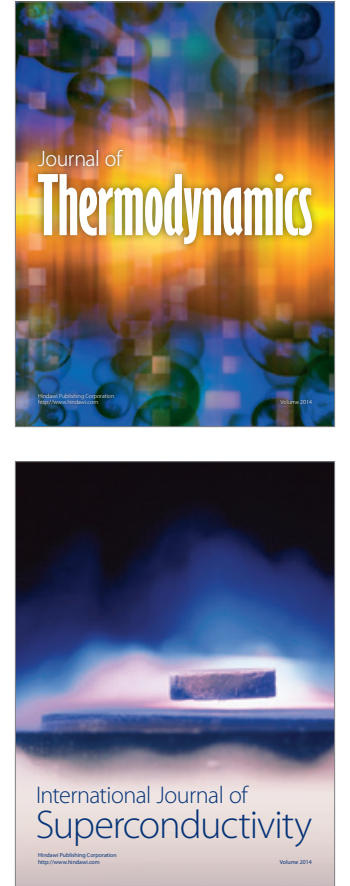
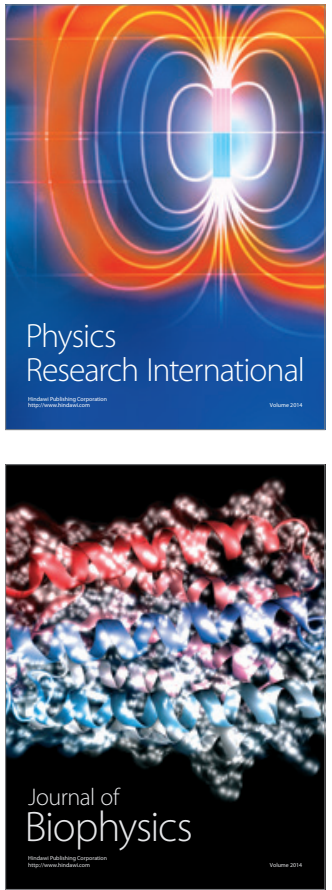
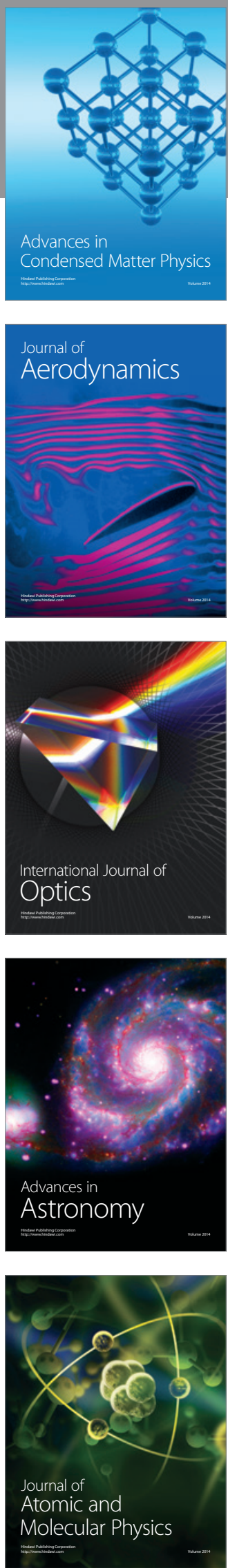